\title{
Estratégias de integração entre atenção primária à saúde e atenção especializada: paralelos entre Brasil e Espanha
}

Strategies for integrating primary health care with specialized care: parallels between Brazil and Spain

Patty Fidelis de Almeida', Juan Gérvas², José-Manuel Freire³ ${ }^{3}$ Lígia Giovanella² $^{2}$

1 Doutora em Saúde Pública pela Escola de Saúde Pública Sérgio Arouca, da

Fundação Oswaldo Cruz (ENSP/FIOCRUZ)

- Rio de Janeiro (RJ), Brasil. Professora e Pesquisadora do Centro de Ciências da Saúde da Universidade Federal do Recôncavo da Bahia (UFRB) - Cruz das Almas (BA), Brasil.

patty@ufrb.edu.br

${ }^{2}$ Escuela Nacional de Sanidad, Instituto de Salud Carlos III.

jgervasc@meditex.es

${ }^{3}$ Escuela Nacional de Sanidad, Instituto de Salud Carlos III.

jmfreire@isciii.es

${ }^{4}$ Doutora em Saúde Pública pela Escola de Saúde Pública Sérgio Arouca, da Fundação Oswaldo Cruz (ENSP/FIOCRUZ)

- Rio de Janeiro (RJ), Brasil. Pesquisadora do Núcleo de Estudos Político-Sociais em Saúde do NUPES/DAPS/ENSP/FIOCRUZ

giovanel@ensp.fiocruz.br
RESUMO: Este artigo analisa estratégias desenvolvidas por Brasil e Espanha para integrar Atenção Primária à Saúde e Especializada. Entre as iniciativas comuns, observa-se: papel de filtro exercido pelo médico de família, territorialização dos serviços de saúde, especialistas consultores/matriciamento e adoção de protocolos clínicos consensuados. No Brasil, ressalta-se a recente implantação de sistemas descentralizados de regulação, e, na Espanha, a já consolidada informatização da história clínica em APS. A criação de história clínica única é um desafio para ambos os países. Iniciativas que promovam maior relação interpessoal foram consideradas mais exitosas para integrar profissionais dos dois níveis.

PALAVRAS-CHAVE: Atenção primária à saúde; integração de sistemas; serviços de saúde

ABSTRACT: This paper examines strategies developed by Brazil and Spain for integrating Primary Health Care with Specialized Care. Common measures included: the filter role played by family doctors, territorialization of health services, specialist consultants/matrix support and consensual clinical protocols. Of particular note, in Brazil, is the recent introduction of decentralized regulation systems and, in Spain, the long established computerization of PHC clinical records. Both countries face the challenge of creating unified clinical records. Measures to foster more interpersonal relationship were considered the most successful strategies for integrating health workers from the two levels

KEYWORDS: Primary health care; systems integration; health services 


\section{Introdução}

Espanha e Brasil caracterizam-se pela implantação de sistemas nacionais de saúde com princípios organizativos semelhantes, como financiamento por impostos gerais, cobertura universal e processos de reforma no âmbito da Atenção Primária à Saúde (APS), iniciados nas décadas de 1980 e 1990, respectivamente, em condiçóes socioeconômicas e tradiçóes históricas distintas (quadro 1). Ainda que guiados por princípios comuns, são marcantes as desigualdades de acesso e qualidade entre os serviços públicos de saúde dos dois países. O quadro 1 apresenta outros indicadores que caracterizam diferenças entre os sistemas de saúde brasileiro e espanhol.

No Brasil, a implantação do Sistema Único de Saúde (SUS) representou contexto favorável a processos de reforma do modelo assistencial, no qual se insere o Programa Saúde da Família. Inicialmente formulado como um programa vertical, orientado a populaçóes de risco, em um segundo momento, passou a ser considerado pelo governo brasileiro a principal estratégia de reforma do modelo de atenção em saúde. Além de promover maior acesso à atenção primária, a Estratégia Saúde da Família (ESF) incorporou, entre suas diretrizes, o desenvolvimento de ações de promoção da saúde, prevenção de agravos, maior participação social e intersetorialidade, aliadas à definição de porta de entrada pela APS e à formação de equipe multiprofissional (BRASIL, 2006) (quadros 1 e 2).

O Sistema Nacional de Salud espanhol (SNS), instituído em 1986, pela Ley General de Sanidad, representou, segundo Freire (2004), opção política pela experiência, considerada exitosa, dos serviços nacionais de saúde e uma evolução da Asistencia Sanitaria de la Seguridad Social (ASSS). Originada do Seguro Obligatorio de Enfermedad, criado na década de 1940, o seguro social teve sua cobertura progressivamente ampliada e, desde seu início, instituiu o médico generalista como primeiro contato. Neste sentido, a criação do Sistema Nacional de Salud, em 1986, representou trajetória coerente com o desenvolvimento histórico da previdência social espanhola, cuja cobertura foi se incrementando até a década de 1980.
As pressóes dos profissionais recém-formados na especialidade de Medicina de Família e Comunidade (MF\&C), criada em 1978 (CARRIO; BADÍA, 2008), e a crescente insatisfação da população com os serviços oferecidos motivaram a promulgação do Real Decreto $137 / 1984$, considerado um marco na criaçáo de um novo modelo de APS no país. As principais reformas centraram-se na contrataçẫo de médicos generalistas em dedicação integral, formação de equipes multidisciplinares, melhoria da infraestrutura física, construção de novos Centros de Saúde e fortalecimento da função de gatekeeper do generalista, agora, com formação em MF\&C (FREIRE, 2004, 2005; RICO; FREIRE; GÉRVAS, 2007) (quadros 1 e 2).

Desde 1999, o Sistema Nacional de Salud espanhol é financiado por meio de impostos gerais federais e das dezessete Comunidades Autônomas (CA), que respondem pela gestão e organização de toda a assistência sanitária nos territórios. Os serviços sanitários estão organizados em Áreas de Saúde, com população entre 200 e 250 mil habitantes, e em dois grandes níveis de atenção: o primeiro nível, ou APS, composto por Centros de Saúde e consultórios isolados, geralmente, em meio rural, e o segundo nível: a atenção especializada - prestada por hospitais gerais denominados hospitais de área -, no qual também estáo concentrados os meios diagnósticos e os serviços de urgência 24 horas (ESPAÑA, 2006) (quadro 1).

Passados mais de vinte anos desde o início da reforma da APS, na Espanha, e mais de uma década da implantação da ESF no Brasil, a integração entre Atenção Primária à Saúde e Atenção Especializada (AE), assim como ocorre em outros países, converteu-se em uma preocupaçáo e um desafio a ser enfrentado (HOFMARCHER; OXLEY; RUSTICELLI, 2007; GÉRVAS; RICO, 2005). Segundo Gérvas (2009), a integração entre níveis segue como um tema pendente para o sistema de saúde espanhol. Para o autor, a reforma da APS privilegiou mudanças na carteira de serviços, no tipo e na distribuição dos recursos, em detrimento de ações pró-coordenação, sendo esse o escopo de medidas mais recentes. No Brasil, após período de ampla expansão da ESF, principalmente a partir dos anos 2000, ainda são incipientes, na maior parte dos municípios, 
Quadro 1. Características gerais dos sistemas de saúde - Brasil e Espanha, 2009

\begin{tabular}{|c|c|c|}
\hline CARACTERÍSTICA & BRASIL & ESPANHA \\
\hline $\begin{array}{l}\text { Cobertura pública } \\
\text { populacional }\end{array}$ & Universal (de direito, não de fato) & Universal (de fato, não de direito) \\
\hline Fontes de financiamento & Impostos gerais & Impostos gerais \\
\hline $\begin{array}{l}\text { Gasto total em saúde \% } \\
\text { do PIB }\end{array}$ & $8,4(2008)(*)$ & $8,5 \%(2007)(* *)$ \\
\hline $\begin{array}{l}\text { Gasto público em saúde } \\
\% \text { do gasto total em } \\
\text { saúde }\end{array}$ & $44,0(2008)(*)$ & $71.8(2007)(* *)$ \\
\hline $\begin{array}{l}\text { Gasto total em saúde per } \\
\text { capita (\$US PPP) }\end{array}$ & $837(2007)(*)$ & $2.671(2007)(* *)$ \\
\hline $\begin{array}{l}\text { \% de população coberta } \\
\text { por seguros privados de } \\
\text { saúde }\end{array}$ & $20,6(2007)\left(^{* * *}\right)$ & $\begin{array}{l}14.4(2006)(*) \text {, sendo } 50 \% \text { deste total perten- } \\
\text { centes ao Seguro Social de Funcionários }\end{array}$ \\
\hline $\begin{array}{l}\text { Entidade político- } \\
\text { territorial responsável } \\
\text { pela gestão e organização } \\
\text { do sistema de saúde }\end{array}$ & $\begin{array}{l}\text { MUNICÍPIOS: responsáveis pela APS - } \\
\text { descentralização finalizada em 2004; parte } \\
\text { dos municípios de maior porte - todo } \\
\text { o sistema de saúde, incluindo atenção } \\
\text { especializada e hospitalar; } \\
\text { ESTADOS: responsáveis pela gestão da média } \\
\text { e alta complexidade, referência para vários } \\
\text { municípios }\end{array}$ & $\begin{array}{l}\text { MUNICÍPIOS: não têm competências na } \\
\text { organização do SNS } \\
\text { COMUNIDADES AUTÔNOMAS - CA: } \\
\text { (estados) responsáveis por todo o sistema de } \\
\text { saúde - descentralização finalizada em } 2002 .\end{array}$ \\
\hline $\begin{array}{l}\text { Entes responsáveis pela } \\
\text { organização do sistema } \\
\text { de saúde }\end{array}$ & $\begin{array}{l}\text { Secretarias Municipais e Estaduais de } \\
\text { Saúde - órgão da administração pública } \\
\text { direta - organização indiferenciada dentro } \\
\text { da administração pública correspondente } \\
\text { (Município, Estado) }\end{array}$ & $\begin{array}{l}\text { Todas as CA têm Serviços Autônomos de } \\
\text { Saúde (Serviço Vasco, Galego, etc.). São } \\
\text { organizações com personalidade jurídica } \\
\text { própria, com diversos formatos (organismos } \\
\text { autônomos, empresas públicas, entes } \\
\text { públicos de direito privado) }\end{array}$ \\
\hline $\begin{array}{l}\text { Organização territorial- } \\
\text { populacional do sistema } \\
\text { de saúde }\end{array}$ & $\begin{array}{l}\text { REGIÕES OU DISTRITOS DE SAÚDE: em } \\
\text { construção com formatos diversos } \\
\text { ÁREAS DE SAÚDE: para as Equipes de Saúde } \\
\text { da Família (até } 4.000 \text { hab) }\end{array}$ & $\begin{array}{l}\text { ÁREAS DE SAÚDE: } 200.000 \text { a } 250.000 \text { hab. } \\
\text { ZONAS BÁSICAS DE SAÚDE: } 5.000 \text { a } 25.000 \\
\text { hab para a APS }\end{array}$ \\
\hline $\begin{array}{l}\text { Níveis de organização da } \\
\text { atenção em saúde }\end{array}$ & $\begin{array}{l}3 \text { Níveis: } \\
\text { ATENÇÃO BÁSICA (APS) } \\
\text { ATENÇÃO DE MÉDIA COMPLEXIDADE } \\
\text { especializada ambulatorial e hospitalar } \\
\text { ATENÇÃO DE ALTO CUSTO/ALTA } \\
\text { COMPLEXIDADE ambulatorial e hospitalar }\end{array}$ & $\begin{array}{l}2 \text { Níveis: } \\
\text { ATENÇÃO PRIMÁRIA À SAÚDE } \\
\text { ATENÇÃO ESPECIALIZADA: ambulatorial } \\
\text { hospitalar e internação hospitalar }\end{array}$ \\
\hline
\end{tabular}




\begin{tabular}{|c|c|c|}
\hline CARACTERÍSTICA & BRASIL & ESPANHA \\
\hline $\begin{array}{l}\text { Prestadores de serviços } \\
\text { do sistema público de } \\
\text { saúde }\end{array}$ & $\begin{array}{l}\text { APS: própria pública em Centros de Saúde } \\
\text { ATENÇÃO ESPECIALIZADA: pública e privada, } \\
\text { 35\% dos procedimentos realizados pelo SUS } \\
\text { foram realizados por prestadores privados (\#) } \\
\text { ATENÇÃO HOSPITALAR: privada e pública, } \\
55 \% \text { das internações realizadas pelo SUS } \\
\text { foram em prestadores privados (\#) }\end{array}$ & $\begin{array}{l}\text { APS: própria pública em Centros de Saúde } \\
\text { ATENÇÃO ESPECIALIZADA/HOSPITALAR: } \\
70 \% \text { prestadores públicos próprios (varia } \\
\text { segundo CA) }\end{array}$ \\
\hline $\begin{array}{l}\text { Vínculo laboral dos } \\
\text { trabalhadores sanitários } \\
\text { públicos }\end{array}$ & $\begin{array}{l}\text { Diversos regimes: servidores públicos, } \\
\text { Regime CLT, contratos temporários }\end{array}$ & $\begin{array}{l}\text { Regime especial de emprego público } \\
\text { ("estatutário") para a grande maioria }\end{array}$ \\
\hline $\begin{array}{l}\text { Médicos por } 10.000 \\
\text { habitantes }\end{array}$ & $17,4(2007)\left(^{* * *}\right)$ & $36,5(2007)(* *)$ \\
\hline $\begin{array}{l}\text { Enfermeiros por } 10.000 \\
\text { habitantes }\end{array}$ & $9,4(2007)(* * *)$ & $75,4(2007)\left(^{* *}\right)$ \\
\hline $\begin{array}{l}\text { Leitos hospitalares por } \\
10.000 \text { habitantes }\end{array}$ & $18.8(2010)\left(^{* * *}\right)$ & $33(2006)(* *)$ \\
\hline $\begin{array}{l}\text { Número de consultas } \\
\text { médicas por habitante/ano }\end{array}$ & 2,6 (SUS) $(2007)\left({ }^{* * *}\right)$ & $8,1(2006)\left({ }^{* *}\right)$ \\
\hline
\end{tabular}

Fonte: *WHO Statistical Information System (WHOSIS), 2010;

** OECD Health Data, 2009

*** Indicadores e Dados Básicos, 2008

\# Ministério da Saúde - DATASUS

medidas para alcançar melhor coordenação. A ausência de integração da rede assistencial, associada à oferta insuficiente, repercute negativamente no acesso aos serviços especializados, considerados o grande gargalo do SUS (ALMEIDA et al., 2010).

Estudos identificam um conjunto de reformas capazes de potencializar a capacidade dos sistemas de saúde de melhorar a coordenação dos cuidados, que pode ser definida como a articulação entre os diversos serviços e açóes relacionados à atenção em saúde, de forma que, independentemente do local onde sejam prestados, estejam sincronizados e voltados ao alcance de um objetivo comum (ALMEIDA et al. 2010; HOFMARCHER; OXLEY; RUSTICELLI, 2007; BOERMA, 2007; NUNEEZ; LORENZO; NAVARRETE, 2006). $O$ presente artigo identifica estratégias de coordenação dos cuidados por meio da análise de instrumentos de integração entre Atenção Primária à Saúde e Atenção Especializada, desenvolvidas em municípios brasileiros e Comunidades Autônomas espanholas. Estudos demonstram que a coordenação dos cuidados sustenta-se na existência de uma rede integrada de prestadores de serviços de saúde, de modo que distintas intervençóes sejam percebidas e experenciadas pelo usuário de forma contínua e adequada às suas necessidades de atenção em saúde (ALMEIDA et al. 2010; HOFMARCHER; OXLEY; RUSTICELLI, 2007; BOERMA, 2007; NUNEEZ; LORENZO; NAVARRETE, 2006). Deste modo, além de colaborar para maior conhecimento teórico e prático no campo da coordenação dos cuidados, aqui compreendida como essencial à oferta de uma APS abrangente, objetiva-se identificar práticas exitosas que, tendo em vista especificidades de cada país, possam constituir lições aprendidas. 
Quadro 2. Características da Atenção Primária à Saúde - Brasil e Espanha, 2009

\begin{tabular}{|c|c|c|}
\hline INDICADOR & BRASIL & ESPANHA \\
\hline $\begin{array}{l}\text { Tradição - antecedentes } \\
\text { de Medicina Geral }\end{array}$ & $\begin{array}{l}\text { Medicina Previdenciária com ações } \\
\text { especializadas de pronto-atendimento, sem } \\
\text { tradição em MF\&C } \\
\text { Centros de Saúde Pública para controle de } \\
\text { endemias e atendimento a grupos específicos } \\
\text { por meio de ações programáticas } \\
\text { Poucas iniciativas locais em Medicina Geral e } \\
\text { Comunitária }\end{array}$ & $\begin{array}{l}\text { Medicina de Assistência Pública Domiciliar } \\
\text { (século XIX) } \\
\text { Anos 40: o Seguro Social inicia com a } \\
\text { Medicina e Pediatria geral (médicos de } \\
\text { cabecera) }\end{array}$ \\
\hline $\begin{array}{l}\text { Processo de reforma da } \\
\text { APS }\end{array}$ & $\begin{array}{l}\text { Iniciado em } 1994 \text { (criação do Programa Saúde } \\
\text { da Família) }\end{array}$ & $\begin{array}{l}\text { Iniciado em } 1984 \text { (RD137/84) Criação das } \\
\text { Equipes de Atenção Primária }\end{array}$ \\
\hline $\begin{array}{l}\text { APS porta de entrada } \\
\text { (filtro) }\end{array}$ & $\begin{array}{l}\text { Proposta a partir de } 1994 \text { em áreas cobertas } \\
\text { pela Estratégia Saúde da Família }\end{array}$ & $\begin{array}{l}\text { Sim, desde os anos de 1940, início do Seguro } \\
\text { Social }\end{array}$ \\
\hline $\begin{array}{l}\text { Co-pagamento por } \\
\text { consultas }\end{array}$ & Não & Não \\
\hline $\begin{array}{l}\text { Territórios } \\
\text { geograficamente } \\
\text { definidos para a APS }\end{array}$ & $\begin{array}{l}\text { Somente para áreas cobertas pela ESF: áreas e } \\
\text { micro-áreas de saúde }\end{array}$ & $\begin{array}{l}\text { Sim: Zonas Básicas de Saúde (Centros de } \\
\text { Saúde) }\end{array}$ \\
\hline $\begin{array}{l}\text { Composição das Equipes } \\
\text { de APS }\end{array}$ & $\begin{array}{l}\text { Médico generalista, enfermeiro e auxiliar de } \\
\text { enfermagem, agentes comunitários de saúde } \\
\text { e equipe de saúde bucal (odontólogo, ACD e/ } \\
\text { ou THD) }\end{array}$ & $\begin{array}{l}\text { Médico de Família e Comunidade, Pediatra, } \\
\text { Enfermeiras, Administrativos }\end{array}$ \\
\hline $\begin{array}{l}\text { Lista de população por } \\
\text { médico de família }\end{array}$ & $\begin{array}{l}\text { Não (população adscrita a Equipe de Saúde } \\
\text { da Família e não ao médico de família - de } \\
3.000 \text { a } 4.000 \text { hab/ESF ) }\end{array}$ & $\begin{array}{l}\text { Sim: entre } 1.300 \text { e } 1.800 \text { pessoas registradas } \\
\text { por médico de família }\end{array}$ \\
\hline $\begin{array}{l}\text { Livre eleição do médico/ } \\
\text { equipe de Saúde da } \\
\text { Família }\end{array}$ & Não & $\begin{array}{l}\text { Sim (possibilidade de mudar de médico de } \\
\text { família voluntariamente) }\end{array}$ \\
\hline $\begin{array}{l}\text { Sistema de pagamento } \\
\text { dos médicos }\end{array}$ & Salário & $\begin{array}{l}\text { Salário + pequeno complemento por } \\
\text { capitação }\end{array}$ \\
\hline $\begin{array}{l}\text { Horário normal de } \\
\text { funcionamento dos } \\
\text { Centros de Saúde }\end{array}$ & $8: 00-17: 00$ (segunda a sexta-feira) & $\begin{array}{l}08: 00-21: 00 h \text { (segunda a sexta-feira) em dois } \\
\text { turnos }\end{array}$ \\
\hline $\begin{array}{l}\text { Carga horária dos } \\
\text { médicos de APS }\end{array}$ & $\begin{array}{l}\text { 40:00 hs semanais em dois turnos (manhã e } \\
\text { tarde) }\end{array}$ & $\begin{array}{l}35 \text { hs semanais em um turno (manhã ou } \\
\text { tarde) }\end{array}$ \\
\hline $\begin{array}{l}\text { Formação especializada } \\
\text { em Medicina de Família e } \\
\text { Comunidade }\end{array}$ & Não obrigatória & $\begin{array}{l}\text { Atualmente obrigatória } \\
\text { Iniciada } 1978 \text { - } 4 \text { anos de duração, igual as } \\
\text { outras especialidades. }\end{array}$ \\
\hline
\end{tabular}

Fonte: Elaboração Própria 


\section{Método}

Para identificar e analisar as estratégias de integração entre APS e AE, no Brasil e na Espanha, foram analisados dados qualitativos de estudos de caso desenvolvidos em quatro municípios brasileiros (GIOVANELLA; ESCOREL; MENDONÇA, 2009) e em três comunidades autônomas espanholas. Buscou-se analisar características, similaridades e diferenças com relação às estratégias de integração entre níveis assistenciais, na perspectiva de gestores e gerentes da saúde, consideradas elementos fundamentais para alcançar o atributo da coordenação dos cuidados (KRINGOS et al., 2010). Conforme destaca Conill (2006), embora a organização e a distribuição dos serviços sejam distintas entre os países e que seja impossível a simples importação de experiências desenvolvidas em contextos socialmente distintos, a análise comparada permite conhecer diferentes estratégias para o enfretamento dos problemas de saúde, refletir sobre sua utilidade e situar determinado sistema de saúde em uma perspectiva mais ampla.

Os municípios brasileiros foram selecionados de forma intencional, com base em pesquisa anterior, realizada por Escorel et al. (2004), e em entrevistas com informantes-chave do Ministério da Saúde, de modo a escolher experiências consolidadas de implantação da ESF (quadro 3). Neste sentido, as experiências analisadas não podem ser generalizadas para todo o país, que apresenta modelos diferenciados e graus distintos de implantação e cobertura por equipes de saúde da família. Na Espanha, foram selecionadas - com base em entrevistas com informantes-chave da Escuela Nacional

Quadro 3. Critérios de seleção e características dos municípios estudados, 2009

\begin{tabular}{|c|c|c|c|c|}
\hline $\begin{array}{l}\text { CRITÉRIOS/ } \\
\text { CARACTERÍSTICAS }\end{array}$ & ARACAJU & BELO HORIZONTE & FLORIANÓPOLIS & VITÓRIA \\
\hline $\begin{array}{l}\text { Ano de implantação } \\
\text { do PSF }\end{array}$ & 1998 & 2002 & 1998 & 1998 \\
\hline $\begin{array}{l}\text { Cobertura } \\
\text { populacional do PSF } \\
>\text { de } 50 \%\end{array}$ & $86,7 \%$ & $69,6 \%$ & $71,3 \%$ & $60,1 \%$ \\
\hline $\begin{array}{l}\text { Número de ESF } \\
\text { implantadas* }\end{array}$ & $127(128)$ & $484(504)$ & $84(84)$ & $56(62)$ \\
\hline Número de UBS & 50 & 145 & 48 & 30 \\
\hline $\begin{array}{l}\text { Presença de } \\
\text { práticas inovadoras } \\
\text { preliminarmente } \\
\text { identificadas }\end{array}$ & Acolhimento & $\begin{array}{c}\text { Acolhimento } \\
\text { Integração da rede }\end{array}$ & $\begin{array}{c}\text { USF campo de prática } \\
\text { para graduação }\end{array}$ & $\begin{array}{c}\text { Intersetorialidade } \\
\text { Adesão AMQ }\end{array}$ \\
\hline $\begin{array}{l}\text { População municipal } \\
2007\end{array}$ & 505.286 & 2.399 .920 & 406.564 & 317.085 \\
\hline Região do país & Nordeste & Sudeste & Sul & Sudeste \\
\hline $\begin{array}{l}\text { Habilitação em } \\
\text { gestão plena do } \\
\text { sistema municipal }\end{array}$ & Sim & Sim & Não & Não \\
\hline
\end{tabular}


de Sanidad, órgão do Ministério de Ciência e Inovação, que desenvolve programas de formação e pesquisa voltados à melhoria do Sistema Nacional de Saúde espanhol - Comunidades Autônomas com capacidade de 'liderança', cujas mudanças e inovações são de grande influência para as demais, quais sejam: Catalunha, Madri e País Basco. Nos três casos, as unidades de análise foram compostas pela área metropolitana mais importante, respectivamente, Barcelona, Madri e Bilbao. Nos dois países, foram eleitas unidades com capacidade decisória (municípios, no Brasil, e CA, na Espanha).

A principal fonte de informação do estudo foram 81 entrevistas semiestruturadas, realizadas com gestores, gerentes da saúde, profissionais e representantes de entidades médicas e de enfermagem, realizadas, nos municípios, entre os meses de maio e setembro de 2008, e nas CAs, de março a agosto de 2009 (quadro 4). O roteiro de entrevista foi definido com base em categorias de análise utilizadas por Almeida et al. (2010) para identificar e avaliar estratégias de integração entre níveis assistenciais. Os roteiros de entrevista foram adaptados em função das especificidades dos sistemas de saúde de cada um dos países.

As entrevistas semiestruturadas foram gravadas e transcritas. Foi realizada análise de conteúdo, e os resultados organizados segundo as categorias pré-definidas. Os dados passaram por análise vertical de conteúdo, considerando categorias, grupos de informantes-chave, municípios e CAs, e por leitura horizontal, comparando-se as perspectivas do conjunto de atores envolvidos. Além das categorias pré-definidas, novos elementos foram identificados com base em temas emergentes nas entrevistas. A apresentação dos resultados está estruturada em quatro dimensôes: posição ocupada pela APS e prioridade do tema da integração entre níveis de atenção nas políticas locais de saúde; instrumentos de integração dos serviços de saúde; organização dos fluxos para atenção especializada; e cultura de colaboração entre APS e AE (quadro 5).

Quadro 4. Composição da amostra de informantes, Brasil e Espanha, 2009

\begin{tabular}{|c|c|c|c|}
\hline GRUPOS DE INFORMANTES & BRASIL & ESPANHA & TOTAL \\
\hline \multicolumn{4}{|c|}{ GESTORES } \\
\hline Secretário Municipal de Saúde & 4 & & 4 \\
\hline Gestor de Área de APS & & 5 & 5 \\
\hline \multicolumn{4}{|c|}{ GERENTES/PROFISSIONAIS/ENTIDADES REPRESENTATIVAS } \\
\hline Atenção Básica/ESF & 4 & & 4 \\
\hline Regulação/Controle/Avaliação & 16 & & 16 \\
\hline Vigilância em Saúde & 4 & & 4 \\
\hline Centro de Saúde & 16 & & 16 \\
\hline Diretor médico, de enfermagem e de investigação & & 14 & 14 \\
\hline Diretor clínico de hospital & & 2 & 2 \\
\hline $\begin{array}{l}\text { Médico de Família/enfermagem/representantes de } \\
\text { entidades profissionais }\end{array}$ & 10 & 6 & 16 \\
\hline TOTAL & 54 & 27 & 81 \\
\hline
\end{tabular}


Quadro 5. Características e estratégias de integração entre Atenção Primária à Saúde e Atenção Especializada segundo gestores e gerentes Brasil e Espanha, 2009

\begin{tabular}{|c|c|c|}
\hline DIMENSÕES & BRASIL & ESPANHA \\
\hline \multicolumn{3}{|c|}{ POSIÇÃO OCUPADA PELA APS E PRIORIDADE DO TEMA DA INTEGRAÇÃO ENTRE NÍVEIS DE ATENÇÃO } \\
\hline \multicolumn{3}{|c|}{ NAS POLÍTICAS LOCAIS DE SAÚDE } \\
\hline $\begin{array}{l}\text { Presença do tema no } \\
\text { debate da política de } \\
\text { saúde }\end{array}$ & Sim & Sim \\
\hline $\begin{array}{l}\text { Grau de importância } \\
\text { atribuído }\end{array}$ & Alto & Alto \\
\hline $\begin{array}{l}\text { Capacidade de } \\
\text { intervenção dos gestores } \\
\text { de APS na implementação } \\
\text { de medidas pró- } \\
\text { coordenação }\end{array}$ & $\begin{array}{l}\text { Alta - nas Coordenações de AB/ESF } \\
\text { centralizada na Secretaria Municipal de Saúde }\end{array}$ & Baixa - nas Coordenações locais de APS \\
\hline $\begin{array}{l}\text { Grupos mais afetados por } \\
\text { inadequada coordenação }\end{array}$ & $\begin{array}{l}\text { Crianças, idosos, crônicos e pacientes com } \\
\text { patologias graves com dificuldades para } \\
\text { acessar os serviços de saúde }\end{array}$ & $\begin{array}{l}\text { Crônicos, idosos, gestantes, pacientes } \\
\text { com necessidade de cuidados paliativos, } \\
\text { imigrantes }\end{array}$ \\
\hline $\begin{array}{l}\text { Porta de entrada do } \\
\text { sistema de saúde }\end{array}$ & Unidades de Saúde da Família & Centros de Saúde \\
\hline $\begin{array}{l}\text { Papel de filtro para } \\
\text { atenção especializada }\end{array}$ & Médicos da Equipe de Saúde da Família & $\begin{array}{l}\text { Médicos de Família e Pediatras das Equipes } \\
\text { de Atenção Primária }\end{array}$ \\
\hline \multicolumn{3}{|c|}{ INSTRUMENTOS DE INTEGRAÇÃO DOS SERVIÇOS DE SAÚDE } \\
\hline $\begin{array}{l}\text { História clínica } \\
\text { informatizada em APS }\end{array}$ & Incipiente & Sim (em todos os Centros de Saúde) \\
\hline História clínica única & $\begin{array}{l}\text { Incipiente (entre atenção primária e } \\
\text { especializada) }\end{array}$ & $\begin{array}{l}\text { Não (existência de repositório comum de } \\
\text { informações clínicas) }\end{array}$ \\
\hline $\begin{array}{l}\text { Retorno das informações } \\
\text { clínicas por meio de } \\
\text { instrumento de contra- } \\
\text { referência }\end{array}$ & Irregular, com graus distintos de qualidade & Irregular, com graus distintos de qualidade \\
\hline
\end{tabular}

\section{Resultados}

\section{INTEGRAÇÃO ENTRE ATENÇÃO PRIMÁRIA E ESPE-} CIALIZADA NO BRASIL

\author{
Posição ocupada pela APS e prioridade do tema \\ da integraçấo entre níveis de atençáo nas políticas \\ locais de saúde
}

A integração entre níveis assistenciais tem sido um tema presente no debate da política local de saúde nos quatro municípios brasileiros, e considerada, pelos gestores entrevistados, relevante e necessária para a construção de uma atenção integral em saúde, ainda que medidas para efetivá-la ainda não estejam plenamente implantadas. Há um entendimento de que somente a expansão da Estratégia Saúde da Família não seja suficiente para garantir a integralidade do cuidado, exigindo a articulação com os 


\begin{tabular}{|c|c|c|}
\hline DIMENSÕES & BRASIL & ESPANHA \\
\hline \multicolumn{3}{|c|}{ ORGANIZAÇÃO DOS FLUXOS PARA ATENÇÃO ESPECIALIZADA } \\
\hline $\begin{array}{l}\text { Percurso mais comum do } \\
\text { paciente para acesso à } \\
\text { atenção especializada }\end{array}$ & $\begin{array}{l}\text { Encaminhamento do médico da equipe de } \\
\text { saúde da família }\end{array}$ & $\begin{array}{l}\text { Encaminhamento do médico de família/ } \\
\text { pediatra/ginecologista }\end{array}$ \\
\hline $\begin{array}{l}\text { Circuitos preferenciais } \\
\text { para referência }\end{array}$ & $\begin{array}{l}\text { Priorização de risco indicada pelo médico de } \\
\text { família e avaliada por equipe de reguladores } \\
\text { da SMS }\end{array}$ & $\begin{array}{l}\text { Sim - para suspeita de malignidade e } \\
\text { consultas preferenciais a critério da avaliação } \\
\text { do médico de família }\end{array}$ \\
\hline $\begin{array}{l}\text { Estruturas de APS para } \\
\text { atendimento à urgência }\end{array}$ & $\begin{array}{l}\text { Não - Unidades de Pronto Atendimento } \\
\text { pertencentes à } \mathrm{AE}\end{array}$ & $\begin{array}{l}\text { Sim - Pontos de Atenção Continuada e } \\
\text { Centros de Urgência em APS }\end{array}$ \\
\hline $\begin{array}{l}\text { Articulação entre APS } \\
\text { e serviços de pronto- } \\
\text { atendimento }\end{array}$ & Insuficiente & Insuficiente \\
\hline Guias de prática clínica & $\begin{array}{l}\text { Sim - por patologias e ciclos de vida e } \\
\text { protocolos para definição de fluxos e } \\
\text { regulação para atenção especializada }\end{array}$ & $\begin{array}{l}\text { Sim - por patologias (artrite, hipertrofia } \\
\text { benigna de próstata, insuficiência cardíaca, } \\
\text { etc) }\end{array}$ \\
\hline $\begin{array}{l}\text { Monitoramento das } \\
\text { referências realizadas } \\
\text { pelos profissionais de APS } \\
\text { para AE }\end{array}$ & Não & Não \\
\hline \multicolumn{3}{|c|}{ CULTURA DE COLABORAÇÃO ENTRE APS E AE } \\
\hline $\begin{array}{l}\text { Conhecimento do } \\
\text { trabalho desenvolvido em } \\
\text { APS pelos profissionais } \\
\text { de AE }\end{array}$ & Baixo & Insuficiente, embora com mudanças recentes \\
\hline $\begin{array}{l}\text { Reconhecimento } \\
\text { profissional e social dos } \\
\text { profissionais de APS }\end{array}$ & $\begin{array}{l}\text { Pouco reconhecimento por parte dos demais } \\
\text { especialistas; iniciativas municipais de } \\
\text { valorização dos profissionais de APS }\end{array}$ & $\begin{array}{l}\text { Insuficiente reconhecimento por parte dos } \\
\text { demais especialistas, maior por parte dos } \\
\text { usuários; situação atual mais favorável }\end{array}$ \\
\hline $\begin{array}{l}\text { Iniciativas que favoreçam } \\
\text { conhecimento pessoal } \\
\text { e intercâmbio de } \\
\text { experiências entre APS } \\
\text { e AE }\end{array}$ & $\begin{array}{l}\text { Tele-consulta e tele-conferência; } \\
\text { territorialização dos serviços de AE; } \\
\text { "matriciamento" com interconsulta, sessões } \\
\text { clínicas e capacitações }\end{array}$ & $\begin{array}{l}\text { Especialistas "consultores" com sessões } \\
\text { clínicas e interconsonsulta; deslocamento } \\
\text { dos especialistas ao Centro de Saúde para } \\
\text { atendimento clínico; criação de blog para } \\
\text { profissionais de APS, AE e gestores }\end{array}$ \\
\hline \multicolumn{3}{|c|}{ PRINCIPAIS DIFICULDADES PARA IMPLANTAÇÃO DE ESTRATÉGIAS PRÓ-COORDENAÇÃO } \\
\hline $\begin{array}{l}\text { Principais dificuldades } \\
\text { para implantação } \\
\text { de estratégias pró- } \\
\text { coordenação }\end{array}$ & $\begin{array}{l}\text { Insuficiente integração entre prestadores } \\
\text { municipais e estaduais; baixa qualidade } \\
\text { dos processos de regulação (justificativas } \\
\text { clínicas); insuficiente oferta de atenção } \\
\text { especializada; ausência de políticas para } \\
\text { a média complexidade por parte do } \\
\text { Ministério da Saúde; pouco conhecimento } \\
\text { dos processos de trabalho em APS e } \\
\text { de reconhecimento dos profissionais; } \\
\text { rotatividade de profissionais }\end{array}$ & $\begin{array}{l}\text { Grande independência e ausência de } \\
\text { objetivos comuns entre níveis assistenciais; } \\
\text { insuficiente articulação entre gestores de } \\
\text { APS e AE; aumento do trabalho burocrático } \\
\text { executado pelos médicos; falta de incentivos } \\
\text { para desempenho de ações de coordenação; } \\
\text { baixa institucionalidade e falta de } \\
\text { continuidade das estratégias implementadas; } \\
\text { pouco conhecimento dos processos de } \\
\text { trabalho em APS }\end{array}$ \\
\hline
\end{tabular}


demais níveis do sistema e com as políticas públicas. Ainda assim, se reconhece que a implantação da ESF, na medida em que objetiva organizar a porta de entrada, impulsionou o debate em torno da necessidade de organização da rede, regulação e coordenaçấo dos cuidados. A possibilidade de redução do número de internaçóes hospitalares, de melhoria do acompanhamento do uso de medicamentos e do autocuidado, do fluxo de informaçóes entre serviços e dos ganhos de eficiência do sistema em geral seriam resultados, em médio prazo, de medidas pró-coordenação. Os grupos avaliados como mais afetados por inadequada coordenação foram crianças, mulheres, idosos, pacientes crônicos e com patologias graves que apresentam dificuldades para acessar o sistema de saúde. No entanto, destacou-se que os diversos grupos populacionais necessitam de cuidados contínuos, associados à existência de serviços de saúde integrados (quadro 5).

As equipes de Saúde da Família (SF) estão formalmente definidas como a porta de entrada para o sistema de saúde, embora o papel de filtro, que inclui a referência aos demais níveis, seja exercido pelo médico generalista nas cidades estudadas (quadro 5). Além de porta de entrada, os serviços de APS são identificados pelos gestores e gerentes como o nível de atenção que teria o papel de coordenação dos cuidados no interior do sistema de saúde:

\section{Todo o restante da rede é suporte para a atenção básica (gestor de Belo Horizonte).}

Segundo os entrevistados, a principal estratégia para organização da porta de entrada nas Unidades de Saúde da Família (USF) é o acolhimento, que busca articular atendimento às demandas espontânea e programada, embora não haja garantias explícitas de tempos máximos para atendimento.

\section{Instrumentos de integração dos serviços de saúde}

Um dos principais instrumentos para integrar a APS à atenção especializada é a implantaçẫo de centrais informatizadas de regulação e marcação de procedimentos especializados - SISREG - nas Unidades de Saúde da Família. Mesmo que em fases distintas de implantação entre os casos, os sistemas informatizados vêm permitindo aos gestores conhecer o tamanho real das filas de espera, monitorá-las, definir prioridades clínicas, conhecer o índice de abstenção a consultas e exames, além de garantir maior imparcialidade no controle das agendas.

A implantação da história clínica informatizada nos serviços de atenção primária e especializada ainda é recente e bastante incipiente. Belo Horizonte e Florianópolis, no ano de 2008, estavam em processo de informatização dos prontuários nas USF, com desenvolvimento de história clínica compartilhada com os serviços municipais de especialidades. Nestas cidades, desde o início da implantação, trabalhou-se pela criação de história clínica única entre atenção primária e especializada (quadro 5).

Foi relatada existência de instrumentos formais para referência e contrarreferência. Contudo, a maior parte dos gestores e gerentes afirmou que a contrarreferência não é uma prática comum, o que compromete a continuidade dos cuidados. Entre os motivos assinalados, aparecem a vinculação histórica do usuário com a atenção especializada, o isolamento entre profissionais dos dois níveis, a desqualificação do trabalho do generalista e as dificuldades dos profissionais para registro de dados clínicos.

\section{Organizaçáo dos fluxos para atençáo especializada}

O percurso mais comum do usuário para acesso à atenção especializada, de acordo com os fluxos formais estabelecidos, acontece por meio de encaminhamento dos serviços de APS nos quatro casos. A marcação é realizada com base na referência do médico das equipes de SF (quadro 5). O pedido é inserido no SISREG, e a data e o serviço, em geral, são, posteriormente, informados pelo telefone, pelo Agente Comunitário de Saúde, ou o próprio usuário retorna ao Centro de Saúde para obter a informação. Outra forma de acesso à atenção especializada é a procura direta por unidades de urgência e pronto atendimento. Em todos os municípios, foi recorrente o discurso de que muitos casos que chegam a esses serviços poderiam ser atendidos nas USF. De acordo com avaliação dos entrevistados, a possibilidade de atendimento no mesmo dia e uma certa facilidade de acesso a meios diagnósticos são 
alguns dos fatores explicativos da procura dos serviços de urgência como primeiro contato.

Nos municípios estudados, o sistema de marcação e regulação presente nas USF permite a inserção da referência solicitada pelo médico de família segundo classificação de risco. Em Belo Horizonte, por exemplo, os casos considerados prioritários são avaliados por equipes de médicos reguladores da Secretaria Municipal de Saúde, que definem aqueles que não podem estar na fila de espera, independentemente das cotas físicas de cada Unidade.

Para a organização dos fluxos, são adotadas guias de prática clínica em diversas áreas do cuidado, que, embora sigam recomendaçóes gerais do Ministério da Saúde, passaram por processos de discussão e revisóes locais, com participação de profissionais, gestores e entidades representativas. Em Belo Horizonte, Florianópolis e Vitória, além das guias tradicionais, com base nos ciclos de vida e agravos, foram adotados protocolos para definição de fluxos e regulação da atenção especializada. O processo de construção coletiva dos protocolos funcionou, também, como estratégia de capacitação e interlocução entre profissionais de APS e AE.

Não foi referida prática sistemática de análise dos encaminhamentos realizados pelas equipes de SF por parte do gestor municipal. Contudo, a implantação de sistemas informatizados de regulação para atenção especializada, ao possibilitar o monitoramento on line das filas de espera, vem permitindo o acompanhamento do percurso do usuário e o redimensionamento da oferta dos recursos especializados, de acordo com critérios epidemiológicos e sociais, além de algumas medidas para diminuição dos tempos de espera, como compra de serviços da rede privada (quadro 5).

\section{Cultura de colaboração entre Atençáo Primária à Saúde e Atençáo Especializada}

A preocupação em criar mecanismos para aproximação entre profissionais da APS e da AE, com vistas a criar uma cultura de colaboração entre níveis, ainda é incipiente nos municípios investigados, sendo mais presente em Belo Horizonte. Estratégias de teleconsulta e teleconferência foram consideradas medidas que aproximam profissionais dos dois níveis (quadro 5). Embora constituam importante instrumento para melhorar a integração entre serviços de saúde, segundo avaliação de alguns entrevistados:

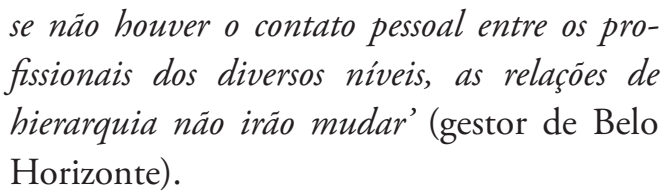

Avaliou-se que o especialista precisa sair do consultório e conhecer diretamente as pressōes e demandas da população, o que possibilitaria mudanças de atitude, de valorização e respeito pelos profissionais que atuam em outros níveis do sistema. O baixo prestígio social e profissional dos trabalhadores de APS agrava a distância em relação às demais especialidades. Na perspectiva de membros de entidades representativas de Medicina de Família e Comunidade, o maior problema enfrentado seria a baixa valorização do médico generalista pelos pares.

A criação de serviços territorializados de atenção especializada, como vem ocorrendo em Belo Horizonte e Florianópolis, que funcionam como referência para as USF de determinado distrito ou regional de saúde, além de aumentar o acesso mais oportuno a consultas e exames de média complexidade, oportuniza maior contato entre generalistas e especialistas e potencializa a resolutividade da APS:

\section{É dentro desta lógica que estamos trabalhando, ou seja, ampliar o acesso à média complexidade é também qualificar a atenção primária (ges- tor de Florianópolis).}

O 'matriciamento' destacou-se entre as inovaçóes implantadas para reduzir o distanciamento e aproximar especialistas e profissionais de APS, além de trabalhar as dificuldades encontradas pelas equipes de SF em seu próprio espaço de trabalho:

Não podemos ter dois modelos, um que seja de atenção básica e outro que não seja. A atenção especializada precisa qualificar a básica. O usuário precisa pertencer a todos os niveis (gestor de Vitória). 
O matriciamento prevê que especialistas apoiem profissionais de APS por meio de interconsulta, discussão de casos clínicos, capacitaçóes, além de funcionar como referência para atendimentos.

\section{INTEGRAÇÃO ENTRE ATENÇÃO PRIMÁRIA E ESPE- CIALIZADA NA ESPANHA}

\section{Posiçáo ocupada pela APS e prioridade do tema da integraçáo entre níveis de atenção nas políticas locais de saúde}

A integração entre níveis assistenciais foi considerada um desafio fundamental nas discussões e políticas de saúde nas três CAs espanholas, e elemento chave para melhoria da qualidade da atenção, ainda que as medidas implantadas nem sempre sejam as mais adequadas. Foi consenso entre os entrevistados que a população a ser atendida é a mesma, a depender da necessidade de saúde em dado momento, e que os serviços são interdependentes.

O grande número de portadores de patologias crônicas vem gerando desafios assistenciais, tanto para a APS quanto para a $\mathrm{AE}$, sendo uma das motivaçôes para o desenvolvimento de açóes pró-coordenação como a melhor integração entre níveis assistenciais. Além disso, a preocupação com o tema foi relacionada a problemas como aumento das listas de espera, repetição de provas diagnósticas e o consequente aumento dos gastos para o sistema sanitário (quadro 5). Entre os grupos mais afetados por inadequada coordenação, foram mencionados portadores de patologias crônicas, idosos, gestantes e aqueles com necessidade de cuidados paliativos. De acordo com avaliação dos entrevistados, grupos com menor acesso às informaçóes sobre a organização do sistema de saúde e aos recursos econômicos são prejudicados por inadequada coordenação, como, por exemplo, imigrantes.

$\mathrm{Na}$ Espanha, o profissional médico com especialidade em Medicina de Família \& Comunidade tem função de filtro para a atenção especializada. A grande maioria dos espanhois utiliza seu médico de família e, também, o pediatra do Centro de Saúde como serviço de procura regular. $\mathrm{O}$ usuário é vinculado a um médico de família e pode, mediante justificativa, solicitar mudança para a lista de outro profissional. Embora a APS tenha passado por reformas nos anos de 1980, que aumentaram seu protagonismo no interior do Sistema Nacional de Salud, desde 1940, a função de porta de entrada já era exercida pela APS. Mais recentemente, em alguns casos, os enfermeiros que compóem as Equipes de Atenção Primária (EAP) passaram a realizar atendimento de primeiro contato, embora a função de filtro seja exercida por médicos de família.

O mais frequente é que não haja nenhum tempo de espera para a APS e que todos sejam atendidos no mesmo dia. Em Bilbao, há uma diretriz para toda Comarca, parte da certificação de qualidade dos serviços, que determina a existência de vagas nas agendas para atendimento dos casos agudos. Apesar do amplo acesso à APS, cujas unidades funcionam em dois turnos, foi relatado que as longas listas de espera para algumas especialidades e a existência de uma certa cultura, que considera a atenção hospitalar como de melhor qualidade, são elementos que levam a população a buscar atendimento diretamente na rede de urgência.

Gestores de Madri destacaram a baixa capacidade de intervenção das gerências de APS na definição de estratégias para melhorar a integração entre níveis assistenciais. Ainda assim, as principais iniciativas em curso, frequentemente, são empreendidas por esse nível de atenção. Uma das razóes citadas seria a de que a atenção primária é quem se responsabiliza e mantém maior vínculo com os usuários, construído por anos de atuação de um mesmo profissional no mesmo Centro de Saúde:

(...) a atenção especializada, em geral, é para
episódios (...). Entretanto, o paciente permane-
ce, ao longo do tempo e de sua vida, longitudi-
nalmente, com a APS. Entáo, muitas vezes, a
necessidade de coordenaçáo parte mais da nossa
necessidade do que da atençáo especializada
(gerente de Bilbao).

\section{Instrumentos de integraçáo dos serviços de saúde}

Desde a década de 1990, foi iniciado o processo de informatização das histórias clínicas em APS, com ritmo diferenciado entre as CAs. Já a rede hospitalar apresenta 
processo mais recente de informatização (quadro 5). Não há história clínica única, compartilhada entre APS e AE. Barcelona e Bilbao optaram pelo desenvolvimento de um 'repositório comum de informações clínicas', que permite aos profissionais de ambos os níveis acessar e compartilhar dados de interesse comum, como exames complementares, diagnósticos, informes de alta hospitalar, contatos com serviços de saúde, tratamentos, entre outros. Essa ferramenta é recente e ainda não está plenamente implantada em todas as unidades de atenção primária e especializada.

A troca de informaçóes clínicas entre níveis de atenção ainda acontece, predominantemente, por meio de guias de referência e contrarreferência náo informatizadas. Na maior parte das vezes, o documento que contém dados clínicos e justificativa para referência é conduzido pelo usuário ao especialista, que deve fazer o registro do atendimento nesse documento. $\mathrm{O}$ processo de retorno das informaçóes clínicas, desde a AE à APS, foi avaliado como irregular, apresentando problemas com relaçáo à qualidade dos registros.

\section{Organização dos fluxos para atençáo especializada}

O percurso mais comum do usuário para obter acesso à atenção especializada ocorre por referência realizada por médicos de família ou pediatras que atuam nos Centros de Saúde (quadro 5). Quando esses profissionais avaliam a necessidade de encaminhamento, preenchem uma guia, na qual dados pessoais, medicaçóes e outras informaçóes podem ser extraídas da história clínica informatizada e acrescidas da justificativa para a referência. A solicitação é conduzida pelo usuário ao funcionário administrativo, que acessa a agenda de consultas e exames disponíveis, segundo o hospital de área. Assim, o agendamento da consulta com especialista é realizado pelo Centro de Saúde, e a data e o horário, em geral, são informados nesse momento.

Mais recentemente, foi relatada criação de circuitos especiais de referência para casos que, segundo a avaliação do médico de família, não poderiam entrar em filas de espera e não têm perfil para atendimento de urgência. Em Madri, esses circuitos estão estabelecidos para casos de 'suspeita de malignidade'. Desse modo, o usuário deve ser comunicado sobre a disponibilidade de consulta especializada em até 48 horas e atendido em até 15 dias; e para 'consultas preferenciais', que representam situaçóes intermediárias entre a urgência e o circuito normal de referência, devendo ser avaliadas em até um mês. Nas demais CAs, os circuitos preferenciais também privilegiaram casos de suspeita de câncer. Contudo, foi mencionado que tais circuitos enfrentam problemas para sua operacionalização, como a realização de referências inadequadas:

Se gerou mais demora diagnóstica, apesar da criação de circuitos especiais de referência para pacientes com suspeita de câncer. O que ocorre é que se encaminha todo mundo, os que podem ou não ter câncer, se produzindo um gargalo ainda maior (gestor de Madri).

Para os episódios que acontecem fora do horário de atendimento dos Centros de Saúde, estão disponíveis Pontos de Atenção Continuada (PAC). Esses serviços fazem parte da APS e estão voltados ao atendimento de casos sem perfil para urgências hospitalares.

Guias de prática clínica para agravos específicos também têm representado estratégia utilizada para organização de fluxos entre atenção primária e especializada. Em Bilbao e Barcelona, foi relatada elaboração conjunta desses instrumentos, uma vez que implica a definição de atividades coordenadas entre os dois níveis (quadro 5).

O número de encaminhamentos e as listas de espera estão disponíveis para as gerências de atenção primária, embora não tenha sido relatada prática sistemática de avaliação desses dados. Conforme gestores e gerentes de Madri, não é possível saber o número de pacientes que realizaram consulta ou exame especializados, informação disponível apenas nos hospitais e enviada às direções de APS, esporadicamente. Foi considerado que mais que um problema de oferta insuficiente, as longas filas de espera, para algumas especialidades, decorreriam da falta de objetivos comuns entre atenção primária e especializada

Ter objetivos comuns nos ajudaria. (...) Há recursos que não são econômicos. Por isso, temos 
que encontrar a fórmula de nos coordenarmos mais facilmente (gerente de Madri).

\section{Cultura de colaboraçáo entre Atençáo Primária à Saúde e Atenção Especializada}

De forma geral, avaliou-se como insuficiente o conhecimento dos profissionais que atuam na atenção especializada sobre o processo de trabalho na APS, situação que vem se modificando, positivamente, ao longo do tempo (quadro 5). Foi consenso que um maior conhecimento mútuo poderia gerar mudanças positivas na relação entre profissionais dos dois níveis:

\section{(...) quando os especialistas vêm aos Centros de Saúde e passam a conhecer como trabalhamos, se estabelecem relaçóes de confiança e vontade de trabalhar mais em conjunto. Quando ficam somente no hospital, se esquecem de como fun- ciona o todo (gerente de Madri).}

Neste sentido, as iniciativas para favorecer maior conhecimento pessoal e intercâmbio de experiências entre profissionais de APS e AE foram consideradas as estratégias mais exitosas para fortalecer a integração entre níveis:

Conhecer-se fisicamente também é importante. (...) existe a tecnologia... mas o contato pessoal é importante, estar diante do especialista, falar com ele... (gerente de Bilbao).

A distância entre os profissionais também foi atribuída ao fato de que o médico de família não recebe os mesmos reconhecimento e prestígio que os demais especialistas, uma das condiçóes para que possa exercer o papel de coordenação no interior do sistema de saúde:

as estratégias utilizadas são as mesmas utilizadas por todos: protocolizar patologias, definir critérios de referência, utilizar ferramentas conjuntas... Tudo isso não tem utilidade se não parte de uma base: que o profissional de APS tenha o mesmo nivel, responsabilidade e valorização que os demais médicos do sistema de saúde. Todos têm que situar-se no mesmo nivel, com papéis distintos... (médico de família de Bilbao).

Entre as medidas implantadas para aproximar profissionais, destaca-se a experiência de 'especialistas consultores' em áreas como cardiologia, dermatologia, gastroenterologia, oftalmologia, traumatologia, entre outras (quadro 5). Estes profissionais, vinculados a hospitais de referência, se deslocam aos Centros de Saúde para realização de interconsulta, sessões clínicas, capacitaçôes e elaboração conjunta de protocolos:

\section{Uma das vantagens de ter especialistas nos Centros de Saúde é a aproximação, o reconhe- cimento mútuo. Muitas vezes, o especialista atende somente no hospital e maneja só um tipo de paciente. Quando estão nos Centros de Saúde, há uma adaptação a uma forma distin- ta de trabalhar (gerente de Barcelona).}

Em Bilbao, a criação de um blog para troca de informaçôes, artigos, opinióes, realização de sessões clínicas e, sobretudo, como instrumento de comunicação foi mencionada como uma iniciativa para aproximar profissionais e para melhorar a integração entre níveis:

o objetivo é compartilhar uma cultura de que vale a pena trabalhar de forma conjunta, compartilhar conhecimento (gestor de Bilbao).

\section{Discussão}

Nos dois países, considerou-se insuficiente o conhecimento dos profissionais que atuam em serviços especializados sobre o processo de trabalho em APS e a valorização de seus profissionais, o que interfere na criação de uma cultura de colaboração. Situação mais problemática é enfrentada no Brasil, onde a especialidade de Medicina de Família \& Comunidade é de reconhecimento recente. Foi consenso entre os entrevistados espanhois que, além do uso das TIC, são necessárias ações que 
promovam maior contato interpessoal. Esses resultados corroboram os achados de Martinez et al. (2009), em estudo com gestores e profissionais de organizações sanitárias espanholas. Neste país, destacou-se a experiência de 'especialistas consultores'. A territorialização dos serviços de atenção especializada, como ocorre em dois casos investigados, também possibilita maior contato entre profissionais que atuam na mesma região de saúde. Essas intervençóes parecem oportunas na medida em que discussóes com assessores externos têm se mostrado inefetivas para promover mudanças nos processos de referência (GRIMSHAW et al., 2005).

Nas CAs estudadas, foram criados circuitos especiais de referência e outras iniciativas para definição de tempos máximos de espera. Embora tenham sido avaliadas positivamente, referências inadequadas ou tentativas de diminuir tempos de espera por meio de fluxos especiais podem gerar problemas adicionais de desigualdade de acesso e retardar o atendimento em casos necessários (GÉRVAS et al., 2008). Nos municípios brasileiros estudados, o SISREG permite inclusão de casos prioritários, ainda que coexistam problemas de oferta insuficiente de atenção especializada (ALMEIDA et al., 2010). A iniciativa de criar comissões locais em Centros de Saúde e protocolos de regulação objetiva qualificar as referências, promover maior discussão dos casos e busca de uma segunda opinião. Estudos demonstram que alternativas intermediárias, como uma segunda opinião, no próprio âmbito da APS, apresentam resultados positivos na modificação das taxas de referência (GRIMSHAW et al., 2005).

A definição de protocolos clínicos foi estratégia adotada pelos dois países. As evidências disponíveis indicam que a adoção passiva de diretrizes clínicas, provavelmente, não melhora o processo de referência, salvo quando envolve os diversos prestadores na elaboração e na implantação (GRIMSHAW et al., 2005). Estudo realizado por Martinez et al. (2009) corrobora essa assertiva, destacando que guias e protocolos clínicos podem facilitar acordos sobre a atuação dos profissionais somente se consensuados.

$\mathrm{Na}$ Espanha, desde os anos de 1990, iniciou-se processo de informatização da história clínica em APS, embora, até o momento, não tenham sido observados avanços no desenvolvimento de uma história única. Ainda assim, a criação de um 'repositório comum de informaçóes clínicas', acessado pela APS e AE, aparece como alternativa. No Brasil, a história clínica informatizada é bastante incipiente. Nos dois países, a maior parte da contrarreferência apresenta graus distintos de qualidade e regularidade, problema histórico e persistente, que reflete a insuficiente comunicação entre níveis (REIG et al., 2004).

Em síntese, experiências desenvolvidas por Brasil e Espanha apresentam potencialidades para melhorar a integração da rede. Entre as iniciativas comuns, com graus distintos de desenvolvimento, observa-se: papel de filtro exercido pelo médico de família no sistema de saúde espanhol, e, no Brasil, nas áreas cobertas pela ESF; territorialização dos serviços de saúde; estratégias para aproximar profissionais e qualificar as açóes de APS, como os 'especialistas consultores' e o 'matriciamento'; e adoção de protocolos clínicos consensuados. Nos municípios brasileiros investigados, ressalta-se a recente implantação de sistemas descentralizados de regulação que permitem acompanhar o percurso dos usuários e as filas de espera. $\mathrm{Na}$ Espanha, destaca-se a consolidada informatização da história clínica em APS. A criação da história clínica única é um desafio colocado aos dois países. Em ambos, é reconhecida a necessidade de políticas e investimentos para valorizaçáo dos trabalhadores e do cuidado prestado no campo da APS, bem como da promoçấo de maior contato pessoal e de relaçóes diretas entre profissionais, com vistas à criação de uma cultura de colaboração, sendo essas as iniciativas consideradas mais exitosas para integração entre atenção primária e especializada.

\section{Referências}

ALMEIDA, P.F. et al. Desafios à coordenação dos cuidados em saúde: estratégias de integração entre níveis assistenciais em grandes centros urbanos. Cadernos de Saúde Pública, v.26, n.2, 2010. p.286-298.
BOERMA, W.G.W. Coordination and integration in European primary care. In: SALTMAN, R.S.; RICO, A.; BOERMA, W.G.W. (orgs.). Primary care in the driver's seat? Organizational reform in Europe- 
an primary care. Berkshire: Open University Press, 2007. p. 3-21.

BRASIL. Ministério da Saúde. Secretaria de Atenção à Saúde. Departamento de Atenção Básica. Política nacional de atenção básica. Brasília: Editora Ministério da Saúde, 2006.

CARRIO, F.B.; BADÍA, J.G. La atención primaria española en los albores del siglo XXI. Gestión Clínica y Sanitaria, v.10, n.1, 2008. p. 3-7.

CONILL, E. Sistemas comparados de saúde. In: CAMPOS, G.W.S. et al. (orgs.). Tratado de Saúde Coletiva. São Paulo,Rio de Janeiro: Editora Hucitec, Editora Fiocruz, 2006. p. 563-613.

ESPAÑA, Ministerio de Sanidad y Consumo. Dossier nacional de atención primaria en salud y la integración con otros niveles de atención. Madrid: EuroSocial Salud, 2006. Disponível em: <http:// www4.ensp.fiocruz.br/eventos_novo/dados/arq6973.doc> Acesso em: 24 jul. 2009.

FREIRE, J.M. La atención primaria de la salud y los hospitales en el Sistema Nacional de Salud. In: NAVARRO LÓPEZ, V. (coord.). El Estado de Bienestar en España. Madrid: Tecnos, 2004. p. 239-291.

La cobertura poblacional del Sistema Nacional de Salud: importancia y retos de la universalización y la equidad en el aseguramiento. In: REPULLO, J.R.; OTEO OCHOA, L.A. Un nuevo contrato social para un Sistema Nacional de Salud sostenible. Madrid: Ariel, 2005. p. 61-99.

FREIRE, JM; J, GÉRVAS, J. La coordinación entre Atención Primaria y Especializada: Reforma del sistema sanitario o reforma del ejercicio profesional? Revista de Administración Sanitaria,v. 4, n. 2, 2006. p. 357-382.

GÉRVAS, J. Atención primaria: 1984-2007. La práctica clínica (acceso y proceso). In: NAVARRO, V.; MARTíN-ZURRO, A. (orgs.) La atención primaria de salud en España y sus comunidades. Barcelona: SEMF\&C, 2009. p. 91-104.

GÉRVAS, J. et al. Paradojas en la derivación de primaria a especializada. Atención Primaria, v. 40, n. 5, 2008. p. 253-255.

GÉRVAS, J.; RICO, A. La coordinación en el sistema sanitario y su mejora a través de las reformas europeas de la atención primaria. SEMERGEN, v. 31, n. 9, 2005. p.418-423.

GIOVANELLA, L.; ESCOREL, S.; MENDONÇA, M.H.M. (coords.) Estudos de caso sobre a implementação da estratégia saúde da família em grandes centros urbanos. Relatório final. Rio de Janeiro: ENSP/Fiocruz, 2009. Disponível em: <http://www4.ensp.fiocruz. br/biblioteca/home/exibedetalhesBiblioteca.cfm?ID=9439\&tipo $=B>$. Acesso em: 24 set. 2009 .
GRIMSHAW, J.M. et al. Interventions to improve outpatient referrals from primary care to secondary care. The Cochrane Database of Systematic Reviews, v. 3, 2005. Art. No.: CD005471. DOl: 10.1002/14651858.CD005471.

HOFMARCHER, M.M.; OXLEY, H.; RUSTICELLI, E. Improved Health System performance through better care coordination. Health Working Paper n. 30. Paris: OECD, 2007. Disponível em:<http:// www.oecd.org/dataoecd/22/9/39791610.pdf>. Acesso em: 20 mar. 2009.

KRINGOS, D.S. et al. The breadth of primary care: a systematic literature review of its core dimensions. BMC Health Services Research, v. 10, n. 65, 2010. Disponível em: <http://www.biomedcentral. com/1472-6963/10/65>. Acesso em: 23 may 2010.

MARTÍNEZ, D.H; NAVARRETE, M.L.V.; LORENZO, I.V. Factores que influyen en la coordinación entre niveles asistenciales según la opinión de directivos y profesionales sanitarios. Gaceta Sanitaria, v. 23, n. 4, 2009. p. 280-286.

NÚÑ̃EZ, R.T.; LORENZO, I.V.; NAVERRETE, M.L. La coordinación entre niveles asistenciales: una sistematización de sus instrumentos y medidas. Gaceta Sanitaria, v. 20, n. 6, 2006. p.485-495.

REIG, B. Evaluación de la calidad del documento de interconsulta. ¿Influye la adecuada cumplimentación del médico de familia en la respuesta que obtiene del especialista? Atención Primaria, v. 34, n. 6, 2004. p. 300-305.

RICO, A.; FREIRE, J.M.; GÉRVAS, J. El sistema sanitario español (19762006): factores de éxito en perspectiva internacional comparada. In: ESPINA, A. (coord.). Estado de Bienestar y competitividad. La experiencia europea. Madrid: Fundación Carolina/Siglo XXI, 2007. p. 401-444.

Recebido para publicação em agosto de 2012

Versão definitiva em novembro de 2013

Suporte financeiro: Ministério da Saúde

Conflito de interesse: inexistente 